\title{
Lack of direct evidence for natural selection at the candidate thrifty gene locus, PPARGC1A
}

Murray Cadzow ${ }^{1,2}$, Tony R. Merriman ${ }^{1,2}$, James Boocock ${ }^{1,2}$, Nicola Dalbeth ${ }^{4}$, Lisa K. Stamp ${ }^{5}$, Michael A. Black ${ }^{1,2}$, Peter M. Visscher ${ }^{2,6,7}$ and Phillip L. Wilcox ${ }^{1,2,3,8^{*}}$

\begin{abstract}
Background: The gene PPARGC1A, in particular the Gly482Ser variant (rs8192678), had been proposed to be subject to natural selection, particularly in recent progenitors of extant Polynesian populations. Reasons include high levels of population differentiation and increased frequencies of the derived type 2 diabetes (T2D) risk 482Ser allele, and association with body mass index (BMI) in a small Tongan population. However, no direct statistical tests for selection have been applied.
\end{abstract}

Methods: Using a range of Polynesian populations (Tongan, Māori, Samoan) we re-examined evidence for association between Gly482Ser with T2D and BMI as well as gout. Using also Asian, European, and African 1000 Genome Project samples a range of statistical tests for selection ( $F_{S T}$, integrated haplotype score $(\mathrm{iHS})$, cross population extended haplotype homozygosity (XP-EHH), Tajima's $D$ and Fay and Wu's $H$ ) were conducted on the PPARGCTA locus.

Results: No statistically significant evidence for association between Gly482Ser and any of BMI, T2D or gout was found. Population differentiation $\left(F_{\text {ST }}\right)$ was smallest between Asian and Pacific populations (New Zealand Māori $\leq 0$. 35, Samoan $\leq 0.20$ ). When compared to European (New Zealand Māori $\leq 0.40$, Samoan $\leq 0.25$ ) or African populations (New Zealand Māori $\leq 0.80$, Samoan $\leq 0.66$ ) this differentiation was larger. We did not find any strong evidence for departure from neutral evolution at this locus when applying any of the other statistical tests for selection. However, using the same analytical methods, we found evidence for selection in specific populations at previously identified loci, indicating that lack of selection was the most likely explanation for the lack of evidence of selection in PPARGC1A.

Conclusion: We conclude that there is no compelling evidence for selection at this locus, and that this gene should not be considered a candidate thrifty gene locus in Pacific populations. High levels of population differentiation at this locus and the reported absence of the derived 482Ser allele in some Melanesian populations, can alternatively be explained by multiple out-of-Africa migrations by ancestral progenitors, and subsequent genetic drift during colonisation of Polynesia. Intermediate 482Ser allele frequencies in extant Western Polynesian populations could therefore be due to recent admixture with Melanesian progenitors.

Keywords: Natural selection, Selective sweeps, Thrifty gene, Gout, Type 2 diabetes, BMI

\footnotetext{
* Correspondence: phillip.wilcox@otago.ac.nz

${ }^{1}$ Department of Biochemistry, University of Otago, Dunedin, New Zealand

${ }^{2}$ Virtual Institute of Statistical Genetics (www.visg.co.nz), Dunedin, New

Zealand

Full list of author information is available at the end of the article
} 


\section{Background}

The thrifty gene theory is based on the hypothesis that alleles causing increased weight, insulin resistance and type 2 diabetes (T2D) in contemporary populations were an adaptation to fluctuating food availability [1], for example from extreme environmental events and extended sea travel, in ancestral populations. Any genuine thrifty gene variant would have been selectively advantageous and therefore subject to selection during human evolution. Thrifty genetic variants arising early in hominid evolution, for example the uricase knockout [2], would be expected to be monomorphic and shared by all human populations. However, candidate thrifty variants arising more recently in human evolution and polymorphic in contemporary populations should exhibit signatures of selection in the immediate genomic vicinity.

The PPARGC1A transcriptional regulator has a central role in insulin signalling and mitochondrial regulation [3]. The functional Gly482Ser (rs8192678) variant (reviewed in [4]) in the PPARGC1A gene is associated with body mass index (BMI) in a Tongan (Polynesian) population [4]. This led to the hypothesis that the Gly482Ser variant represents a candidate 'thrifty variant' in Pacific populations. This was based largely on the observation that the postulated thrifty (derived) 482Ser allele exhibited the highest prevalence world-wide in Polynesian populations, who also have high levels of T2D and BMI. In contrast, low T2D prevalence levels have been recorded in Papuan populations lacking the 482Ser allele [5]. This 482Ser allele observation also supported by a previous report of extreme $F_{\mathrm{ST}}$ values (a measure of inter-population difference at specific genetic variants) at the PPARGC1A locus [5]. To date however, this hypothesis has not been formally tested in a range of Polynesian populations using more direct assessments of natural selection.

The Māori and non-indigenous Polynesian populations of Aotearoa New Zealand (NZ) have a high prevalence of obesity, T2D and related metabolic conditions such as gout [6-8]. The reasons for this appear to be complex and are the result of a combination of poor environmental dietary exposure (contributed to by socioeconomic status) and inherited genetic variants [9]. However, extremely little is known about the etiology of obesity and T2D in NZ Māori and Pacific. If the PPARGC1A gene were associated with weight and subject to selection in Pacific populations, then this would be important knowledge on the aetiology of these conditions in contemporary populations. Furthermore, evidence for selection during Polynesian population history would aid in the destigmatisation of obesity. Here, we tested for association between Gly482Ser rs8192678, BMI, T2D and gout in several Polynesian populations. We also examined evidence for recent positive selection at the derived 482Ser allele and in the region of PPARGC1A, by applying a recently developed analytical pipeline [10] to genomic data from the various Polynesian and 1000 Genomes Project world-wide populations.

\section{Methods \\ Populations}

New Zealand (NZ) Māori, Cook Island Māori, Samoan, Tongan and NZ Caucasian sample sets were examined (Table 1). Samples were collected as part of the "Genetics

Table 1 Populations, genotyping platforms and analyses used to evaluate evidence of association and selection in PPARGC1A

\begin{tabular}{|c|c|c|c|c|}
\hline & & Phenotype & & \\
\hline Population & Genotyping Platform & Gout $(n)$ & Control $(n)$ & Analysis \\
\hline NZ Caucasian & TaqMan SNP Assay & 647 & 360 & 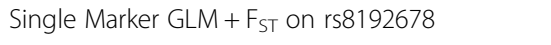 \\
\hline NZ Māori & TaqMan SNP Assay & 137 & 176 & Single Marker GLM + F $\mathrm{ST}$ on rs8192678 \\
\hline Cl Māori & TaqMan SNP Assay & 71 & 64 & 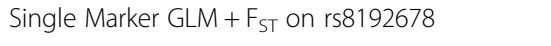 \\
\hline Samoan & TaqMan SNP Assay & 144 & 76 & Single Marker GLM + F $\mathrm{ST}$ on rs8192678 \\
\hline Tongan & TaqMan SNP Assay & 73 & 41 & 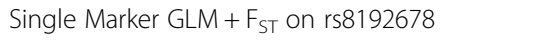 \\
\hline Māori & AXIOM SNP chip & 35 & 36 & 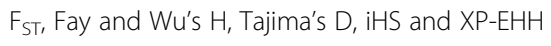 \\
\hline \multirow[t]{2}{*}{ Samoan } & OMNlexpress chip & 48 & 48 & 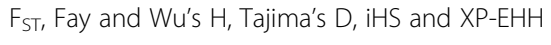 \\
\hline & & \multicolumn{3}{|l|}{ Unknown Phenotype } \\
\hline $1 \mathrm{KGP}$ CEU & WGS & 99 & & F $_{\text {ST, }}$ Fay and Wu's H, Tajima's D, iHS and XP-EHH \\
\hline $1 \mathrm{KGP}$ CHB & WGS & 103 & & F $_{\text {ST, }}$ Fay and Wu's H, Tajima's D, iHS and XP-EHH \\
\hline $1 \mathrm{KGP}$ CHS & WGS & 108 & & F $_{\text {ST, }}$ Fay and Wu's H, Tajima's D, iHS and XP-EHH \\
\hline 1KGP GBR & WGS & 92 & & $F_{S T}$, Fay and Wu's H, Tajima's D, iHS and XP-EHH \\
\hline $1 K G P Y R I$ & WGS & 109 & & $\mathrm{~F}_{\mathrm{ST}}$, Fay and Wu's H, Tajima's D, iHS and XP-EHH \\
\hline
\end{tabular}

All individuals in the Mãori AXIOM and Samoan OMNlexpress datasets were also genotyped by the TaqMan ${ }^{\circledR}$ assay, and included in their respective population groups. NZ = New Zealand. $\mathrm{Cl}=$ Cook Islands. WGS = whole genome sequencing. 1KGP = 1000 genomes project (http://www.1000genomes.org/home). Populations used were Utah, USA residents with Northern and Western European ancestry (CEU, Han Chinese in Beijing, China (CHB), Southern Han Chinese (CHS), China, British in England and Scotland (GBR) and Yoruba in Ibadan, Nigeria YRI. For a complete description of the 1000 Genomes project samples see http:// www.1000genomes.org/data\#DataAvailable. No phenotypic data were available for these populations 
of Gout in Aotearoa" case-control study [11] and ethical approval was obtained from the New Zealand Multiregion Ethics committee (MEC/105/10/130). Each participant provided informed written consent. Patients with gout satisfied, by clinical examination, the American Rheumatism Association preliminary criteria for acute gout [12]. BMI and self-reported T2D status was recorded at time of recruitment. Subjects were classified into ancestry groups based on self-reported ancestry of grandparents. Table 1 provides the number of samples in each population, and the methods used for genotyping and data analyses. Tables 2 and 3 describe clinical data and ancestry information for the NZ sample sets.

Five additional populations were obtained from the 1000 Genomes Project phase 3 data release (see [13]): two Asian populations: Han Chinese in Beijing, China (CHB), Southern Han Chinese, China (CHS); two of European ancestry: British in England and Scotland (GBR) and Utah residents with Northern and Western European ancestry (CEU); and one of African ancestry: Yoruba in Ibadan, Nigeria (YRI). The $\mathrm{CHB}$ and $\mathrm{CHS}$ populations are intermediate with regard to Gly482Ser frequencies between the African (YRI) and European (GBR, CEU) on the one hand and the Polynesian populations on the other [14].

\section{Genotypic data}

Two genotypic data sets were used for this study: (a) a SNP-specific assay corresponding to a Glycine (C allele) -Serine ( $\mathrm{T}$ allele) substitution at amino acid position 482 in the PPARGC1A gene (dbSNP ID rs8192678 residing on chromosome 4 (GRCh37 chr4:23815662) and genotyped on a subset of the above populations (see Table 1); and (b) chromosome 4-wide genotypic data. For (a) SNP rs8192678 was genotyped with TaqMan $^{\circ}$ assay ID C__1643192_20 (Life Technologies, Carlsbad, CA) using a LightCycler 480 Real-Time PCR System (Roche Applied Science, Indianapolis, IN). The MINI MELT program was run from within the LightCycler software, and genotypes were assigned based on clustering within the software. Clusters were visually checked with misassigned genotypes corrected where appropriate or designated as unknown. 9\% of samples were re-genotyped and cross checked as a quality control measure. All regenotyped samples had complete agreement with the original genotypes. For (b) genotypic data for whole chromosome analyses were obtained either directly from SNP microarrays or whole genome sequencing. For the SNP microarrays, 71 individuals who self-reported four Māori grandparents were genotyped using an Affymetrix AXIOM genome-wide ASI array (Table 1). Similarly, 96 individuals self-reporting four Samoan grandparents were genotyped with an Illumina Human OmniExpress bead chip. The individuals genotyped by SNP microarray were a subset from those genotyped with the Taqman ${ }^{\circ}$ assay. Whole genome sequence (WGS) from 1000 Genomes Project phase 3 data release (via [13]) was used.

\section{Association analyses}

To determine associations between BMI and rs8192678 genotype, single marker linear regressions were undertaken using the $\mathrm{R}$ statistical software environment [15]. Hardy-Weinberg equilibrium exact tests were calculated using the $\mathrm{R}$ package HardyWeinberg [16]. For each population, three modes of gene action were tested: additive, dominant (both $\mathrm{C}$ and derived $\mathrm{T}$ allele), and over-dominant. Age, sex, gout affection status, T2D, and Structure-estimated ancestry proportions (calculated as described in [11]) were included as covariates for BMI. For T2D and gout affection status, logistic regression was performed with the same covariates. A metaanalysis of the Polynesian populations was undertaken by combining the NZ Māori, Cook Island Māori, Tongan and Samoan populations and repeating the marker trait regressions as described above with the added covariate of population.

\section{Selection analyses}

Pairwise $F_{\mathrm{ST}}$ [17] was estimated for all populations for rs8192678. For populations where genome-wide genotypic data were available (i.e., the 1000 Genomes Project and Axiom/Omni-genotyped populations, Table 1), the following statistics were calculated: $F_{\mathrm{ST}}$ between sample sets, Tajima's $D$, Fay and Wu's $H$, and integrated haplotype score (iHS, [18]) for individual populations, and cross population haplotype homozygosity (XP-EHH, [19]) to estimate selection between populations. To calculate these statistics we used a customized analytical pipeline [10]. For these analyses we assumed that the 482Ser allele was the derived allele, based on low frequencies of this allele in African populations [5]. $F_{\mathrm{ST}}$ was calculated for the entire chromosome using the Weir and Cockerham method with negative values manually set to zero [17]. Quantiles of 2.5 and $97.5 \%$ were used to find the most extreme $5 \%$ of values. Tajima's $D[20]$ and Fay and Wu's $H$ [21] were calculated for the whole chromosome using bins of 1,5 and 30 kbp. The Tajima's $D$ for the entire chromosome with thresholds of the 2.5 and $97.5 \%$ quantiles used to establish the most extreme $5 \%$ of values.

The software package selscan [22] was used to calculate iHS and XP-EHH with values for both being normalised in frequency bins genome-wide. Cross population extended haplotype homozygosity was used to detect selection of alleles at or near fixation, and was calculated between populations as described in [19]. An iHS or XP-EHH absolute value of greater than 3.29 was used as a threshold to estimate the most extreme $1 \%$ of 
Table 2 Clinical and genetic information for sample sets used for association analysis with BMI, T2D and gout

\begin{tabular}{|c|c|c|c|c|c|c|c|c|c|c|}
\hline & $\begin{array}{l}\text { obs missing } \\
(n)\end{array}$ & NZ Caucasian & $\begin{array}{l}\text { obs missing } \\
(n)\end{array}$ & NZ Māori & $\begin{array}{l}\text { obs missing } \\
(n)\end{array}$ & Cl Māori & $\begin{array}{l}\text { obs missing } \\
(n)\end{array}$ & Samoan & $\begin{array}{l}\text { obs missing } \\
\text { (n) }\end{array}$ & Tongan \\
\hline Individuals & - & 1007 & - & 322 & - & 136 & - & 223 & - & 114 \\
\hline Gout (\%) & - & 64.3 & 9 & 42.5 & 1 & 52.2 & 3 & 64.6 & - & 64.0 \\
\hline Diabetes (\%) & 10 & 11.5 & 11 & 42.8 & 4 & 26.5 & 6 & 14.3 & 3 & 18.4 \\
\hline Mean age (range) & - & $59.7(18-94)$ & 1 & $50.9(17-82)$ & - & $52.4(18-88)$ & - & $44.3(17-71)$ & - & $42.8(18-79)$ \\
\hline Sex (\% male) & 2 & 76.1 & 1 & 56.8 & - & 61.8 & 2 & 82.1 & - & 86.8 \\
\hline Mean BMI Gly/Gly (range) & 6 & $29.1(17.8-61.6)$ & 1 & $32.3(23.0-42.2)$ & 2 & $35.9(33.1-40.7)$ & - & $37.2(26.0-62.5)$ & - & $34.5(26.3-44.2)$ \\
\hline Mean BMI Gly/Ser (range) & 9 & $29.3(18.8-60.7)$ & 3 & $33.0(21.3-61.7)$ & - & $34.5(21.4-66.7)$ & 3 & $37.7(22.2-93.4)$ & - & $36.5(26.6-48.5)$ \\
\hline Mean BMI Ser/Ser (range) & - & $29.7(19.3-62.9)$ & 3 & $34.2(18.4-65.8)$ & 1 & $36.5(23.0-80.0)$ & 1 & $36.7(20.5-70.3)$ & 1 & $36.8(25.8-70.6)$ \\
\hline $\begin{array}{l}\text { Allele frequency rs } 8192678 \\
\text { Ser allele (Gout; Control) }\end{array}$ & 2 & $0.346(0.332 ; 0.371)$ & 2 & $0.801(0.803 ; 0.798)$ & - & $0.805(0.782 ; 0.836)$ & - & $0.688(0.698 ; 0.671)$ & - & $0.636(0.651 ; 0.61)$ \\
\hline $\begin{array}{l}\text { Allele frequency rs } 8192678 \\
\text { Ser allele (Diabetes; Control) }\end{array}$ & 2 & $0.346(0.366 ; 0.345)$ & 2 & $0.801(0.788 ; 0.807)$ & - & $0.805(0.847 ; 0.786)$ & - & $0.688(0.703 ; 0.689)$ & - & $0.636(0.667 ; 0.622)$ \\
\hline
\end{tabular}


Table 3 Clinical and ancestral information for sample sets used in tests for selection analysis

\begin{tabular}{|c|c|c|c|c|}
\hline & obs missing $(n)$ & Māori AXIOM & obs missing (n) & Samoan OMNlexpress \\
\hline Number & - & 71 & - & 96 \\
\hline Sex (\% male) & - & 64.7 & - & 74 \\
\hline Gout cases (\%) & - & 49.3 & - & 50.0 \\
\hline Mean BMI (range) & 1 & $34.5(22.1$ to 56.1$)$ & - & 36.1 (20.5 to 93.4) \\
\hline Diabetes (\%) & 3 & 21.1 & - & 12.5 \\
\hline Mean grandparent ancestry (range) & - & $0.916(0.625$ to 1.00$)$ & - & $0.997(0.875$ to 1.00$)$ \\
\hline
\end{tabular}

Mean grandparent ancestry is the proportion of grandparents self-reported as belonging to the ethnicity group

$B M I$ body mass index

values from the score distribution. The iHS normalisation conformed to a standard Gaussian distribution for all populations. For XP-EHH, all pairwise comparisons were performed between the groups of populations that had been genotyped with genome-wide SNP arrays or via WGS.

Fourteen combinations of populations and genes (corresponding to six genes) previously reported by Voight et al. [18] as showing evidence of selection were analysed as positive controls to provide insight into the overall power of detecting signatures of selection using the methods described above (Additional file 1: Table S1).

\section{Results}

\section{Association analyses}

Tests for association between BMI and rs8192678 genotype did not reveal any statistically significant association for any of the gene action models (Table 4). Similarly, no relationship was observed between rs8192678 genotype and either gout affection or T2D status, for any of the modes of genetic action (Table 4). There were also no statistically significant associations observed for the meta-analysis of the Polynesian populations between BMI, or gout affection, or T2D status and rs8192678 under any of the modes of genetic action.

\section{Selection analyses \\ Intra-population tests for selection - Tajima's D, Fay and Wu's $H$ and $i H S$}

Estimates of Tajima's $D$ and Fay and Wu's $H$ for windows surrounding the Gly482Ser position, were within +/$2.5 \%$ limits for all populations and window lengths (Tables 5 and 6). Only the two Chinese populations (CHB and $\mathrm{CHS}$ ) had strong positive values for Tajima's $D$ in the $1 \mathrm{kbp}$ region surrounding, suggesting possible balancing selection, however estimates decayed to values close to those of the chromosome average for the 30kbp window size (Table 5), and were well within $+/-2.5 \%$ thresholds. For Tajima's $D$ in $1 \mathrm{~kb}$ windows that overlapped the PPARGC1A-encoding region, all populations had windows that exceeded both +2.5 and $-2.5 \%$ thresholds (Additional file 2: Figure S1c), but for the $5 \mathrm{~kb}$ windows only the Māori population had windows exceeding the lower threshold (Additional file 2: Figure $\mathrm{S} 1 \mathrm{~b}$ ), indicating the possibility of a selective sweep. However, no population had any window in the PPARGC1A genic region exceeding either threshold for the $30 \mathrm{kbp}$ region for Tajima's $D$ (Additional file 2: Figure S1a). Moreover, there did not appear to be a clear pattern within any population that was consistent with a selective sweep: estimates for the different window sizes were typically dispersed around the chromosome mean for each population rather than showing a pattern where values consistently exceeded the chromosome thresholds. Similarly, for Fay and Wu's $H$, no windows overlapping the PPARGC1A region that exceeded $+/-2.5 \%$ thresholds were observed with windows of $30 \mathrm{kbp}$ (Additional file 3: Figure S2a). Only the Samoan population had a window that exceeded the threshold in the PPARGC1A genic region for the $5 \mathrm{~kb}$ windows (Additional file 3: Figure S2b). For the $1 \mathrm{~kb}$ windows, both the Māori and Samoan populations had regions that exceeded lower thresholds, indicating the possibility of an excess of high frequency derived alleles (Additional file 3: Figure S2c). However, for the region containing rs8192678, no window exceeded the $+/-2.5 \%$ thresholds in any population, irrespective of window size (Table 6). Neither of these site frequency spectra-based tests therefore revealed evidence of selection in the regions containing the Gly482Ser substitution.

We also calculated integrated haplotype homozygosity score (iHS) statistics for rs8192678 for each population using a genetic map. The iHS is a statistic to detect evidence of recent positive selection at a locus and is based on the differential levels of linkage disequilibrium surrounding a positively selected allele compared to the other allele at the same position. Only the Samoan population had $|\mathrm{iHS}|>3.29$ when computed using a genetic map (corresponding to the most extreme $1 \%$ of $|\mathrm{iHS}|$ values) (Table 7 , Fig. 1). Plots of iHS +/- $1 \mathrm{Mbp}$ of the PPARGC1A-encoding region showed relatively few locations within this region that exceeded the 3.29 threshold in any of the populations investigated (Fig. 1). 
Table 4 Results from a single marker generalised linear model for rs8192678 and BMI, T2D and gout

\begin{tabular}{|c|c|c|c|c|c|c|c|c|c|c|}
\hline & Allele Freq $(T)$ & HWE & Additive & $P$ value & Dominant C (Gly) & $P$ value & Dominant T (Ser) & $P$ value & Heterozygous Advantage & $P$ value \\
\hline \multicolumn{11}{|c|}{ Linear regression between BMI and rs8192678 under the 4 genetic models. Beta (95\% Cl) } \\
\hline NZ Caucasian & 0.346 & 0.728 & $0.319(-0.198,0.835)$ & 0.227 & $-0.555(-1.618,0.507)$ & 0.305 & $0.350(-0.358,1.058)$ & 0.332 & $0.102(-0.601,0.806)$ & 0.775 \\
\hline NZ Māori & 0.806 & 0.721 & $0.629(-0.848,2.106)$ & 0.403 & $-0.837(-2.588,0.913)$ & 0.347 & $0.263(-3.885,4.412)$ & 0.901 & $-0.831(-2.625,0.964)$ & 0.363 \\
\hline Cl Māori & 0.805 & 1.000 & $0.612(-2.342,3.566)$ & 0.682 & $-0.627(-3.940,2.686)$ & 0.709 & $1.319(-8.733,11.371)$ & 0.795 & $-0.504(-3.886,2.879)$ & 0.769 \\
\hline Samoan & 0.688 & 0.043 & $-0.610(-2.605,1.386)$ & 0.548 & $0.705(-1.757,3.166)$ & 0.573 & $-0.873(-5.749,4.002)$ & 0.724 & $0.487(-1.989,2.964)$ & 0.698 \\
\hline Tongan & 0.636 & 0.310 & $0.755(-0.958,2.468)$ & 0.384 & $-0.895(-3.382,1.592)$ & 0.477 & $1.336(-2.118,4.791)$ & 0.445 & $-0.218(-2.799,2.363)$ & 0.867 \\
\hline Polynesian & 0.747 & 0.925 & $0.345(-0.611,1.300)$ & 0.479 & $-0.408(-1.587,0.770)$ & 0.496 & $0.470(-1.904,2.843)$ & 0.698 & $-0.301(-1.496,0.895)$ & 0.621 \\
\hline \multicolumn{11}{|c|}{ Logistic regression between gout affection and rs8192678. OR (95\% Cl) } \\
\hline NZ Caucasian & 0.346 & 0.728 & $0.846(0.683,1.047)$ & 0.125 & $1.290(0.828,1.996)$ & 0.256 & $0.819(0.612,1.095)$ & 0.180 & $0.916(0.686,1.221)$ & 0.548 \\
\hline NZ Māori & 0.806 & 0.721 & $1.238(0.737,2.111)$ & 0.425 & $0.896(0.488,1.635)$ & 0.721 & $3.644(0.697,28.949)$ & 0.159 & $1.071(0.578,1.977)$ & 0.827 \\
\hline Cl Māori & 0.805 & 1.000 & $0.785(0.361,1.654)$ & 0.529 & $1.126(0.488,2.611)$ & 0.780 & - & - & $0.936(0.397,2.202)$ & 0.879 \\
\hline Samoan & 0.688 & 0.043 & $1.110(0.638,1.933)$ & 0.711 & $1.097(0.543,2.220)$ & 0.796 & $2.328(0.658,8.556)$ & 0.189 & $1.437(0.707,2.975)$ & 0.320 \\
\hline Tongan & 0.636 & 0.310 & $1.073(0.562,2.039)$ & 0.829 & $1.248(0.477,3.310)$ & 0.651 & $1.911(0.543,6.737)$ & 0.308 & $1.991(0.713,5.971)$ & 0.199 \\
\hline Polynesian & 0.747 & 0.925 & $1.149(0.862,1.533)$ & 0.345 & $0.970(0.678,1.385)$ & 0.865 & $2.024(1.005,4.126)$ & 0.049 & $1.170(0.814,1.685)$ & 0.396 \\
\hline \multicolumn{11}{|c|}{ Logistic regression between T2D and rs8192678. OR (95\% Cl) } \\
\hline NZ Caucasian & 0.346 & 0.728 & $1.046(0.767,1.419)$ & 0.774 & $0.953(0.523,1.835)$ & 0.880 & $1.065(0.700,1.630)$ & 0.771 & $1.041(0.686,1.576)$ & 0.850 \\
\hline NZ Māori & 0.806 & 0.721 & $0.768(0.463,1.294)$ & 0.311 & $1.362(0.737,2.495)$ & 0.319 & $0.706(0.181,3.535)$ & 0.636 & $1.299(0.692,2.407)$ & 0.409 \\
\hline Cl Māori & 0.805 & 1.000 & $2.280(0.901,6.410)$ & 0.096 & $0.348(0.111,0.977)$ & 0.054 & $0.647(0.037,20.494)$ & 0.777 & $0.314(0.095,0.910)$ & 0.042 \\
\hline Samoan & 0.688 & 0.043 & $1.307(0.630,2.814)$ & 0.480 & $0.788(0.329,1.886)$ & 0.590 & $2.043(0.316,40.719)$ & 0.527 & $0.904(0.379,2.142)$ & 0.818 \\
\hline Tongan & 0.636 & 0.310 & $1.063(0.477,2.443)$ & 0.882 & $0.961(0.328,2.888)$ & 0.942 & $1.198(0.233,9.316)$ & 0.841 & $1.030(0.342,3.045)$ & 0.957 \\
\hline Polynesian & 0.747 & 0.925 & $1.738(0.768,4.219)$ & 0.200 & $0.911(0.610,1.335)$ & 0.646 & $1.104(0.476,2.901)$ & 0.827 & $0.928(0.617,1.386)$ & 0.716 \\
\hline
\end{tabular}

The marker was assessed under an additive model, dominance models and heterozygous advantage model. Models are adjusted for gout and diabetes affection, sex, age and non - Caucasian ancestry. Estimate (95\% Cl). NZ = New Zealand. $\mathrm{Cl}=\mathrm{Cook}$ Islands. Polynesian is a combination of NZ Māori, Cl Māori, Samoan, and Tongan population groups. HWE = $P$-value for departure from Hardy Weinberg expectations using exact test, Logistic regression for Cl Māori under the dominant $\mathrm{T}$ model could not be performed 
Table 5 Tajima's D at PPARGC1A calculated with window sizes of $1 \mathrm{kbp}, 5 \mathrm{kbp}$, and $30 \mathrm{kbp}$

\begin{tabular}{|c|c|c|c|c|}
\hline & Tajima's D & 2.5\% Quantile & Chromosome Mean & 97.5\% Quantile \\
\hline \multicolumn{5}{|c|}{ Tajima's D value for $1 \mathrm{~kb}$ window containing rs 8192678} \\
\hline CEU & 0.810 & -1.127 & 0.772 & 3.053 \\
\hline $\mathrm{CHB}$ & 2.400 & -0.835 & 1.068 & 3.199 \\
\hline $\mathrm{CHS}$ & 2.566 & -0.835 & 1.104 & 3.218 \\
\hline GBR & 0.924 & -1.091 & 0.817 & 3.068 \\
\hline YRI & -0.445 & -1.193 & 0.233 & 2.606 \\
\hline Māori & -0.080 & -1.083 & 0.886 & 2.996 \\
\hline Samoan & 0.622 & -1.119 & 0.943 & 3.103 \\
\hline \multicolumn{5}{|c|}{ Tajima's D value for $5 \mathrm{~kb}$ window containing rs 8192678} \\
\hline CEU & 1.010 & -1.358 & 1.089 & 3.805 \\
\hline $\mathrm{CHB}$ & 1.729 & -1.068 & 1.538 & 4.168 \\
\hline $\mathrm{CHS}$ & 1.923 & -1.054 & 1.599 & 4.204 \\
\hline GBR & 1.846 & -1.299 & 1.153 & 3.865 \\
\hline YRI & -0.150 & -1.486 & 0.310 & 2.892 \\
\hline Māori & -1.040 & -1.295 & 1.275 & 3.905 \\
\hline Samoan & -0.235 & -1.383 & 1.363 & 4.074 \\
\hline \multicolumn{5}{|c|}{ Tajima's D value for $30 \mathrm{~kb}$ window containing rs 8192678} \\
\hline CEU & 1.480 & -1.711 & 1.489 & 4.052 \\
\hline $\mathrm{CHB}$ & 2.585 & -1.265 & 2.102 & 4.730 \\
\hline $\mathrm{CHS}$ & 2.267 & -1.175 & 2.201 & 4.788 \\
\hline GBR & 1.963 & -1.571 & 1.567 & 4.185 \\
\hline YRI & 0.029 & -1.693 & 0.432 & 2.693 \\
\hline Māori & -1.098 & -1.463 & 1.732 & 4.416 \\
\hline Samoan & -0.299 & -1.698 & 1.857 & 4.616 \\
\hline
\end{tabular}

Mean, $2.5 \%$ quantile and $97.5 \%$ quantile were calculated for chromosome 4 by population

We also evaluated six genes that had previously been reported as exhibiting evidence of selection by Voight et al. (2006) in specific ancestries. Here, there were 14 combinations of genes and populations (Additional file 1: Table S1) since two ancestries - Asian and Caucasian - were each represented by two populations each in this study (CHS and CHB for Asians, and CEU and GBR for Caucasians see Additional file 1: Table S1). Seven of the 14 genepopulation combinations showed evidence of selection in the 1000 Genomes populations samples used for this study (where we define evidence of selection as $>1$ SNP exceeding the $1 \%$ threshold of iHS values, Additional file 1: Table S1). Overall, the analytical methods used in this study detected evidence of selection at half of the loci previously shown to be under selection [18] that were tested.

\section{Inter-population tests for selection - $F_{S T}$ and XP-EHH}

Population differentiation at rs8192678 was investigated by calculating $F_{\mathrm{ST}}$ statistics for all pairwise combinations all populations that had been genotyped with the Taq$\mathrm{Man}^{\circ}$ assay. Derived allele frequencies from the WGS and chip datasets were very similar to those found in
Myles et al. [4] with Polynesian (NZ Māori 0.83, Samoan 0.72), Asian (CHB 0.37, CHS 0.45), European (CEU 0.37, GBR 0.33) and African (YRI 0.04). Results showed strong differentiation between NZ Caucasian and all of the Polynesian populations, with NZ Māori and Caucasian being the most differentiated (0.332, Table 8). Tongan and Samoan populations were less differentiated from the NZ Caucasian population (0.160 and 0.210 respectively). East - West Polynesia differentiation [23] was also apparent, with $F_{\mathrm{ST}}$ between NZ Māori and Samoan (0.033) and NZ Māori and Tongan (0.069) populations greater than NZ Māori and CI Māori (0.000). The Samoan and Tongan populations had relatively small differentiation between them $\left(F_{\mathrm{ST}}=0.003\right)$. Population differentiation was also estimated for the Gly482Ser variants in the genome-wide data sets (Table 9). For the Gly482Ser substitution, Māori were more strongly differentiated than the Samoan population from all of the 1000 Genomes populations, with strongest differentiation occurring in the Yoruban population followed by the European and Chinese populations. A similar but less extreme trend was observed for the Samoan 
Table 6 Fay's and Wu's $\mathrm{H}$ at PPARGC1A calculated using window sizes of $1 \mathrm{kbp}, 5 \mathrm{kbp}$, and $30 \mathrm{kbp}$

\begin{tabular}{|c|c|c|c|c|}
\hline & Fay and Wu's H & 2.5\% Quantile & Chromosome Mean & 97.5\% Quantile \\
\hline \multicolumn{5}{|c|}{ Fay and Wu's H 1 kb window containing rs 8192678} \\
\hline CEU & -0.094 & -2.549 & -0.168 & 0.710 \\
\hline $\mathrm{CHB}$ & -0.106 & -2.504 & -0.174 & 0.691 \\
\hline $\mathrm{CHS}$ & -0.007 & -2.511 & -0.173 & 0.693 \\
\hline GBR & -0.227 & -2.531 & -0.162 & 0.713 \\
\hline YRI & -1.132 & -2.345 & -0.055 & 0.888 \\
\hline Māori & -0.845 & -2.754 & -0.217 & 0.648 \\
\hline Samoan & -0.345 & -2.884 & -0.223 & 0.667 \\
\hline \multicolumn{5}{|c|}{ Fay and Wu's H 5 kb window containing rs 8192678} \\
\hline CEU & -0.689 & -8.467 & -0.839 & 2.018 \\
\hline $\mathrm{CHB}$ & -0.656 & -8.237 & -0.870 & 1.953 \\
\hline $\mathrm{CHS}$ & -0.754 & -8.133 & -0.865 & 1.952 \\
\hline GBR & -0.286 & -8.309 & -0.811 & 2.025 \\
\hline YRI & 0.091 & -7.300 & -0.276 & 2.719 \\
\hline Māori & -3.996 & -9.132 & -1.085 & 1.778 \\
\hline Samoan & -2.342 & -9.468 & -1.117 & 1.859 \\
\hline \multicolumn{5}{|c|}{ Fay and Wu's H 30 kb window containing rs 8192678} \\
\hline CEU & 2.955 & -36.264 & -5.032 & 7.377 \\
\hline $\mathrm{CHB}$ & 3.675 & -35.444 & -5.221 & 7.381 \\
\hline $\mathrm{CHS}$ & 2.617 & -35.894 & -5.187 & 7.302 \\
\hline GBR & 3.433 & -35.387 & -4.867 & 7.472 \\
\hline YRI & -1.989 & -29.990 & -1.657 & 10.606 \\
\hline Māori & -14.842 & -3.826 & -6.509 & 6.379 \\
\hline Samoan & -5.607 & -41.697 & -6.702 & 6.870 \\
\hline
\end{tabular}

Mean, $2.5 \%$ quantile and $97.5 \%$ quantile were calculated for chromosome 4 by population

population: $F_{\mathrm{ST}}$ was highest for the Yoruban population, with decreasing levels of differentiation between European and Chinese populations respectively. Lower $F_{\mathrm{ST}}$ between Samoan and Caucasian populations compared to the Māori and Caucasian populations was observed in both the genome-wide genotyped populations and the TaqMan ${ }^{\circ}$-genotyped populations.

Table $7 \mathrm{iHS}$ and XP-EHH statistics for rs8192678

\begin{tabular}{llllllll}
\hline & CEU & CHB & CHS & \multicolumn{1}{l}{ GBR } & YRI & Mãori & Samoan \\
\hline CEU & $-2.510^{\mathrm{a}}$ & 0.548 & 0.163 & 0.321 & 1.319 & -1.579 & -0.771 \\
CHB & -0.548 & $-1.912^{\mathrm{a}}$ & -1.578 & -0.392 & 0.492 & -2.695 & -1.705 \\
CHS & -0.163 & 1.578 & $-1.775^{\mathrm{a}}$ & -0.008 & 1.090 & -2.226 & -1.205 \\
GBR & -0.321 & 0.392 & 0.008 & $-2.074^{\mathrm{a}}$ & 0.795 & -1.752 & -0.983 \\
YRI & -1.319 & -0.492 & -1.090 & -0.795 & $-^{\mathrm{a}}$ & -2.565 & -1.754 \\
Māori & 1.579 & 2.695 & 2.226 & 1.752 & 2.565 & $-3.101^{\mathrm{a}}$ & 1.163 \\
Samoan & 0.771 & 1.705 & 1.205 & 0.983 & 1.754 & -1.163 & $-3.560^{\mathrm{a}}$ \\
\hline
\end{tabular}

All values are given as XP-EHH with the exception of those denoted by ${ }^{\text {aindicating }}$ integrated haplotype homozygosity scores (iHS). Rs8192678 had a derived allele frequency $<0.05$ in YRI and therefore iHS was not calculated
We also calculated $F_{\mathrm{ST}}$ for $5 \mathrm{Mbp}$ windows surrounding the Gly482Ser location to determine the (genomic) extent of population differentiation (Table 10) over a wider window. Compared with the estimates at the $r s 8192678$ locus itself, $5 \mathrm{Mbp}$ window-based $F_{\mathrm{ST}}$ estimates were lower but still revealed the same trend of most differentiation between the Polynesian and Yoruban populations, with European populations the next most differentiated, and Chinese populations the least differentiated (Table 9). However, $F_{\mathrm{ST}}$ estimates between Mãori and other genome-wide genotyped populations differed little from estimates between Samoan and other populations.

Cross population extended haplotype homozygosity was calculated across chromosome 4 for all combinations of populations that had been resequenced or genotyped genome-wide. There was no combination of populations where XP-EHH estimates exceeded the $|3.29|$ threshold in the PPARGC1A region (Table 7). Cross population extended haplotype homozygosity values were also plotted across the aforementioned region by pairwise population combination (Fig. 1). 


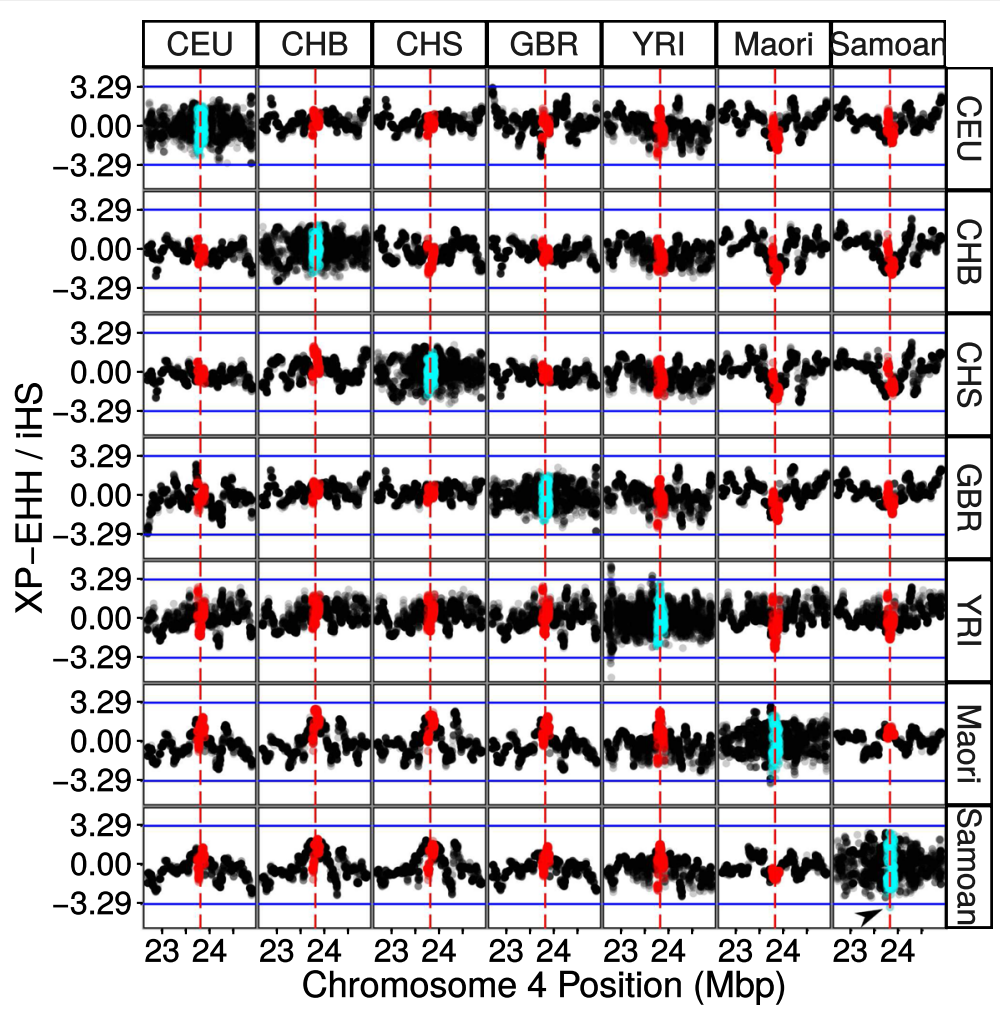

Fig. $1 \mathrm{HHS} / \mathrm{XP}$-EHH by Population. A +/- $1 \mathrm{Mb}$ window centred on PPARGC1A.Threshold of $|3.29|$ for both XP-EHH and iHS is marked with blue lines. Location of PPARGCIA is marked in red for XP-EHH and light blue for iHS. Rs8192678 is indicated by the red dashed line. iHS (within population) is shown on the diagonal. The arrowhead indicates the iHS value for rs 8192678 in Samoans that exceeds the $1 \%$ threshold

\section{Discussion and conclusions}

The primary aim of this study was to directly test the hypothesis that the PPARGC1A locus, in particular the Gly482Ser substitution, has been subject to natural selection in the progenitors of contemporary Polynesian populations. The appropriate basis for concluding a specific gene or region is subject to natural selection has been defined by Vitti et al. [24] as 'A combination of genomic and functional evidence constitutes the current standard for the field'. In this study we combined association analyses between the Gly482Ser genotype and traits either directly (BMI) or indirectly (gout and T2D, which are correlated with BMI) to identify a potential functional role of Gly482Ser. To identify genomic evidence we used a recently-developed analytical pipeline

Table 8 Single marker $F_{\mathrm{ST}}$ for rs8192678

\begin{tabular}{lllll}
\hline & NZ Caucasian & NZ Māori & Cl Māori & Samoan \\
\hline NZ Māori & 0.334 & - & & \\
Cl Māori & 0.324 & 0.000 & - & \\
Samoan & 0.205 & 0.035 & 0.032 & - \\
Tongan & 0.154 & 0.073 & 0.066 & 0.003 \\
\hline
\end{tabular}

$\mathrm{F}_{\mathrm{ST}}$ on the same populations was not calculated and negative $\mathrm{F}_{\mathrm{ST}}$ was set to zero to test selection by a combination of site frequency spectra based statistics, (Tajima's $D$, Fay's and Wu's $H$ ) as well as haplotype-length based measures that examine selection within populations (iHS) or between populations (XP-EHH, [10]). We also estimated population differentiation $\left(F_{\mathrm{ST}}\right)$, which has also been used as an indicator of selection [25], as well as departure from expected HW equilibrium. Of these various approaches, we were able to detect departures from the expected neutral selection model for $F_{\mathrm{ST}}$ only - no other statistics indicated consistent functional or genomic evidence for selection. However, reasons other than selection such as local coancestry can lead to outlier values for $F_{\mathrm{ST}}$ [26] therefore the $F_{\mathrm{ST}}$ results alone are not sufficient to conclude the presence of selection.

Lack of evidence of selection in other statistical tests may be a consequence of lack of power. In this regard, two factors are important in interpreting results from such analyses: (1) the nature of positive selection being investigated, and (2) power of each of the statistical tests to reject the null hypothesis of no departure from neutral model of selection. Regarding (1), two intra-specific selection scenarios were frequently examined: 'hard sweeps' within populations based on selective advantages 
Table 9 Chromosome $4 F_{\text {ST }}$ for rs8192678 in WGS and imputed chip data using 1 bp windows

\begin{tabular}{|c|c|c|c|c|c|c|c|c|}
\hline & Māori & & & & Samoan & & & \\
\hline & $\begin{array}{l}\text { Mean } F_{\mathrm{ST}} \\
\text { (window) }\end{array}$ & $\begin{array}{l}\text { Mean } F_{\mathrm{ST}} \\
\text { (chromosome) }\end{array}$ & $\begin{array}{l}2.5 \% \\
\text { Quantile }\end{array}$ & $\begin{array}{l}97.5 \% \\
\text { Quantile }\end{array}$ & $\begin{array}{l}\text { Mean } F_{\mathrm{ST}} \\
\text { (window) }\end{array}$ & $\begin{array}{l}\text { Mean } F_{\text {ST }} \\
\text { (chromosome) }\end{array}$ & $\begin{array}{l}2.5 \% \\
\text { Quantile }\end{array}$ & $\begin{array}{l}97.5 \% \\
\text { Quantile }\end{array}$ \\
\hline CEU & 0.373 & 0.095 & 0.000 & 0.436 & 0.222 & 0.099 & 0.000 & 0.433 \\
\hline $\mathrm{CHB}$ & 0.348 & 0.062 & 0.000 & 0.320 & 0.199 & 0.044 & 0.000 & 0.216 \\
\hline $\mathrm{CHS}$ & 0.259 & 0.061 & 0.000 & 0.306 & 0.122 & 0.042 & 0.000 & 0.201 \\
\hline GBR & 0.404 & 0.095 & 0.000 & 0.435 & 0.249 & 0.100 & 0.000 & 0.434 \\
\hline YRI & 0.802 & 0.137 & 0.000 & 0.591 & 0.655 & 0.134 & 0.000 & 0.572 \\
\hline
\end{tabular}

arising from a de novo mutation with strong positive effects on fitness, and 'soft sweeps' based on extant variants underpinning heritable characteristics that are typically under polygenic control. Power (i.e., (2), above) to detect evidence of selection for the various methods used here differs depending upon these scenarios [27]. For the 482Ser allele investigated in this study, putative selection could be either via hard or soft sweeps. On the one hand, the previously reported absence of the derived 482Ser allele in some African and New Guinean populations [5] imply selection on a de novo mutation arising prior to ancestors of modern Homo sapiens migrating out Africa. On the other hand however, there is a relative dearth of evidence of hard sweeps in humans [28]. In addition, if PPARGC1A were indeed under selection, it would have been subject to concomitant selection in multiple Polynesian and other populations (e.g., [29]) and acting on extant variation at this locus. This suggests soft sweeps are a more likely scenario, especially as Gly482Ser is possibly one of very many small effect loci impacting the T2D and correlated conditions such as BMI [30, 31].

Of the methods used in this study to directly examine evidence of selection, none indicate selection has possibly occurred at this locus (Tables 4, 5, 6 and 7, and Fig. 1). The power of Tajima's $D$, Fay's and Wu's $H$, and iHS to detect evidence of selection has recently been evaluated by Ferrer-Admetlla et al. [27] for both hard and soft sweeps. For Tajima's $D$ they reported variable power across a wide range of selection coefficients, window sizes, and final (derived) allele frequencies for hard sweeps. In hard sweeps where ending allele frequencies were similar to those observed in Māori populations
(Table 4), moderate-high power was reported, but in soft sweeps power was relatively low $(<0.2)$. Even if such tests are underpowered, the high number of populations, some of which are from the same ancestry (i.e., CEU and GBR being Caucasian, and $\mathrm{CHB}$ and $\mathrm{CHS}$ being Asian) would likely have detected selection should a hard sweep at this locus be occurring. Moreover, it is reasonable to assume that if the 482Ser allele were a genuine thrifty gene variant, selection would be occurring in all populations rather than being restricted to the Polynesian populations, as most ancestral populations would have experienced periodic limitations in food availability. For iHS, Ferrer-Admetlla et al. [27] reported power was generally high (>0.75) irrespective of whether hard or soft sweeps had occurred, and was similarly robust to different ending allele frequencies - which ranged from 0.5 to 0.9 . The derived 482Ser allele frequency estimates in all of the Polynesian sample sets investigated in this study fall within this range.

To provide further insights into whether or not the lack of evidence of selection according to these statistics was due to inadequate sample sizes, we also analysed 14 population/gene combinations that had previously been identified as showing evidence of selection [18]. In our population samples, seven of these 14 combinations had $>1$ SNP exceeding the 1\% iHS threshold, demonstrating that our methods could identify loci previously shown to be under selection (Additional file 1: Table S1). Moreover, in all of the seven cases, multiple SNPs exceeded this threshold, and for two genes, there was evidence of selection in both population samples representing the same ancestry (i.e., evidence of selection in both CEU

Table 10 Chromosome $4 F_{S T}$ for WGS and imputed chip data using 5 Mbp windows

\begin{tabular}{|c|c|c|c|c|c|c|c|c|}
\hline & \multicolumn{4}{|l|}{ Māori } & \multicolumn{4}{|l|}{ Samoan } \\
\hline & $\begin{array}{l}\text { Mean } F_{\mathrm{ST}} \\
\text { (window) }\end{array}$ & $\begin{array}{l}\text { Mean } F_{\mathrm{ST}} \\
\text { (chromosome) }\end{array}$ & $\begin{array}{l}2.5 \% \\
\text { Quantile }\end{array}$ & $\begin{array}{l}97.5 \% \\
\text { Quantile }\end{array}$ & $\begin{array}{l}\text { Mean } F_{S T} \\
\text { (window) }\end{array}$ & $\begin{array}{l}\text { Mean } F_{\mathrm{ST}} \\
\text { (chromosome) }\end{array}$ & $\begin{array}{l}2.5 \% \\
\text { Quantile }\end{array}$ & $\begin{array}{l}97.5 \% \\
\text { Quantile }\end{array}$ \\
\hline CEU & 0.094 & 0.094 & 0.068 & 0.120 & 0.084 & 0.098 & 0.070 & 0.133 \\
\hline $\mathrm{CHB}$ & 0.069 & 0.061 & 0.038 & 0.088 & 0.050 & 0.044 & 0.027 & 0.067 \\
\hline $\mathrm{CHS}$ & 0.067 & 0.060 & 0.035 & 0.090 & 0.047 & 0.042 & 0.025 & 0.067 \\
\hline GBR & 0.088 & 0.094 & 0.067 & 0.124 & 0.082 & 0.099 & 0.070 & 0.133 \\
\hline YRI & 0.145 & 0.138 & 0.106 & 0.178 & 0.142 & 0.136 & 0.098 & 0.188 \\
\hline
\end{tabular}


and GBR populations for $L C T$, and in both $\mathrm{CHB}$ and CHS for SLC445A5, see Additional file 1: Table S1). This indicates that population samples of this size have approximately $50 \%$ power to detect an association in the 1000 Genomes populations - none of which were casecontrol studies - and that when selection is present, there should be multiple SNPs exceeding the threshold. Based on the above results, we therefore expect approximately $\left(1-0.5^{2}\right) \approx 0.75$ probability of at least one of the Samoan and Māori populations used in this study to show evidence of selection using the iHS test. However the (case-control) Samoan and Māori cohorts had (only) one and zero SNPs, respectively, exceeding the 1\% iHS threshold. We also contend that, if anything, the use of a case-control design would be more likely to increase the frequency of haplotypes carrying the selected allele(s), and thus more likely to improve power should this locus be subject to selection. Therefore the lack of a statistically significant difference in 482Ser allele frequencies in the case control Māori cohort used in this study and the previously reported estimate by Myles et al. [5] from a cross-sectional study of a single Mãori tribal group located in the East Coast of the North Island of NZ, is further evidence of the lack of evidence of natural selection at this locus.

A further consideration is the elevated derived 482Ser allele frequency in all non-African populations compared to the African populations $[4,5]$. These show a progressive differentiation of populations at this locus. Thus, if PPARGC1A were a thrifty gene candidate that has been subjected to natural selection, selection would likely have occurred in all non-African ancestral populations due to factors such as climatic extremes, competition with other human groups, and/or the need to adapt to newly colonised environments - all of which are likely to contribute to periodic deprivations in food availability in progenitors of all populations studied.

The highest derived allele frequencies and strongest differentiation from extant African populations occurs in the Cook Islands and NZ Māori populations. Therefore if this locus is indeed a thrifty gene, recent migration histories would suggest that these populations would be likely to show evidence of association and/or selection as ancestors of these populations colonised East Polynesia less than 1500 years ago [32]. Thus we would expect repeated evidence of selection at this locus in most - if not all - of the populations examined. However, the reverse is the case: there is no strong evidence of positive selective at this locus according the iHS statistic, nor Tajima's $D$ nor Fay and Wu's $H$. Similarly, for the NZ Māori population, evidence of possible differential selection indicated by Tajima's $D$ in the PPARGC1A-encoding region exceeding the lower $97.5 \%$ quantile, (Additional file 2: Figure S1a-c) was not reflected in either Fay and Wu's H (Additional file 3: Figure S2a-c) or
iHS (Fig. 1). Moreover, if selection on the derived 482Ser allele were occurring then this would be revealed by XP$\mathrm{EHH}$ - for at least some population pairs. However, none of the XP-EHH exceeded threshold $1 \%$ values in any of the population pairs, nor were there any obvious differences in XP-EHH between pairs of populations that share ancestry (i.e., the CEU - GBR pair, and the CHB CHS pair) compared to population pairs with different ancestries. Further, differences in derived 482Ser allele frequency within Polynesia - particularly those between Western Polynesian (i.e., Tongan and Samoan) and Eastern Polynesian (i.e., NZ and Cook Islands Māori) - are not explained by oceanic voyaging: the geographic distances between Tonga/Samoa and the Cook Islands is significantly less than the Cook Islands and NZ, yet the Cook Islands Māori 482Ser frequency differs from Samoa and Tonga and is virtually identical to NZ Māori. These differences are also reflected in the $F_{\mathrm{ST}}$ values (Table 8 ).

In addition, oral histories of Māori migrations make no reference to extensive loss of life on vaka/waka due to starvation during migration voyages [32], but do describe decisions by tribal groups, whose members were generally closely related (e.g., [33]), to migrate due to food competition and/or ongoing conflicts with other tribal groups - effectively increasing the possibility of genetic drift. We therefore conclude that the lack of evidence for selection in any of the statistical tests used in any of the populations examined in this study is likely an accurate biological reflection for the populations examined rather than a lack of statistical power to detect selective events at this locus with the methods used.

Existing 482Ser allele frequency distributions in the Pacific can be explained by a combination of (a) migration out of Africa by Homo sapiens progenitors and possibly Denisovians who either lacked the 482 Ser allele or lost it via genetic drift (whose descendants include modern-day Melanesians such as Papuans), (b) later migration out of Africa by Homo sapiens progenitors of Polynesians subsequent to the mutation giving rise to the 482Ser allele, which drifted to increasingly higher frequencies in repeated migrations across the Pacific, and (c) followed by subsequent admixture between these Melanesian progenitors with ancestors of modern Polynesians [34]. The higher derived allele frequencies and genetic differentiation in Eastern Polynesians are likely the result of genetic drift in the ancestral population, possibly as a result of founder effects. This model is consistent with the absence of the 482Ser allele in Denisovian DNA sequences; hence any derived populations with Melanesian admixture - such as Tongan populations would have lower frequencies than in Eastern Polynesian populations such as Cook Islands and NZ Māori. Genetic drift therefore could account for the progressive increase in allele frequency in modern non-African human populations, with founder effects arising from successive colonisation of 
islands within Polynesia resulting in the increase in 482Ser allele frequency.

Single marker regression analyses did not reveal any evidence of association between rs8192678 and BMI, gout affection status, or T2D in any of the populations investigated. Although each Polynesian population was small and likely to be underpowered for validating associations between small effect genes and conditions, no associations were found even when Polynesian populations were combined. We also found no evidence for sex-specific effects for any of the three traits in the combined Polynesian populations (data not shown). Our results contrast with those of Myles et al. [4] who reported associations for Tongan populations $(n=184, P=0.014-$ 0.037 ), but are consistent with the lack of association in a NZ Māori tribe (Ngāti Rakaipaaka, $\mathrm{n}=110, P>0.8$ ) that was also reported by Myles et al. [4] as well as a small Tongan population reported by Kimura et al. [14]. The results from these multiple studies when considered together provide no repeated evidence for a functional role of this locus for traits such as T2D, BMI or gout in extant Māori or Samoan populations. However, it is possible that non-additive interaction with unmeasured environmental exposures may obscure evidence for main effect association of Gly482Ser with metabolic phenotypes.

Based on the lack of evidence of association between the Gly482Ser variant with BMI and correlated diseases, as well as lack of evidence of association with any of the tests for selection, we conclude that this study does not support natural selection at either the Gly482Ser variant, or the PPARGC1A region in general. More extreme frequencies of the derived allele in the Polynesian populations can alternatively be explained by genetic drift associated with ancestral population bottlenecks during colonisation. Further, the lack of robust direct candidate gene-based evidence for the thrifty gene hypothesis in light of increasing evidence for selective influence of infectious disease-causing agents on genome composition [35] and other factors such as diet, responses to climate, and skin colour collectively increase doubt regarding ongoing validity of this hypothesis. This is supported by no global of evidence for selection at 65 T2D loci with nominal evidence for selection at individual loci driven by an equal measure of T2D protective and risk haplotypes [31].

\section{Additional files}

Additional file 1: Table S1. Fourteen previously detected combinations of populations and genes from Voight et al. (2006) were analysed using the selscan software package (Szpiech and Hernandez 2014) as positive controls to provide insight into possible power of detection of signatures of selection. Seven of the previously detected 14 associations were repeated, where we defined evidence of selection as > 1 SNP exceeding the 5\% threshold of $\mathrm{iHS}$ values. For two genes ( $L C T$ in both CEU and GBR populations and SLC44A5 in $\mathrm{CHS}$ and $\mathrm{CHB}$ populations) showed evidence of selection in populations with similar ancestry (Caucasian and Asian, respectively). Evidence of selection for one other gene (SNTG1) was observed in only one of two populations with the same ethnicity. (DOCX $25 \mathrm{~kb}$ )

Additional file 2: Figures S1a-c. Tajima's D calculated across chromosome 4 using a $30 \mathrm{kbp}$ sliding window by population. Chromosome 4:22.7-24.9 Mbp is shown with chromosome mean (blue) and 2.5\%, 97.5\% quantiles (purple) from Table 4 marked. Location of PPARGC1A is marked in red. Rs8192678 is marked by a red dashed line. Figure S1b Tajima's D calculated across chromosome 4 using a $5 \mathrm{kbp}$ sliding window by population. Chromosome 4:22.7-24.9 Mbp is shown with chromosome mean (purple) and 2.5\%, 97.5\% quantiles (purple) from Table 4 marked. Location of PPARGC1A is marked in red. Rs8192678 is marked by a red dashed line. Figure S1c Tajima's D calculated across chromosome 4 using a $1 \mathrm{kbp}$ sliding window by population. Chromosome 4:22.7-24.9 Mbp is shown with chromosome mean (blue) and 2.5\%, 97.5\% quantiles (purple) from Table 4 marked. Location of PPARGC1A is marked in red. Rs8192678 is marked by a red dashed line. (ZIP 1473 kb)

Additional file 3: Figures S2a-c. Fay and Wu's $H$ calculated across chromosome 4 using a $30 \mathrm{kbp}$ sliding window. Chromosome 4:22.7-24.9 Mbp is shown with chromosome mean (blue) and 2.5\%, $97.5 \%$ quantiles (purple) from Table 4 marked. Location of PPARGC1A is marked in red. Rs8192678 is marked by a red dashed line. Figure S2b Fay and Wu's H calculated across chromosome 4 using a $5 \mathrm{kbp}$ sliding window. Chromosome 4:22.7-24.9 Mbp is shown with chromosome mean (blue) and $2.5 \%, 97.5 \%$ quantiles (purple) from Table 4 marked. Location of PPARGC1A is marked in red. Rs8192678 is marked by a red dashed line. Figure S2c Fay and Wu's H calculated across chromosome 4 using a 1 kbp sliding window. Chromosome 4:22.7-24.9 Mbp is shown with chromosome mean (blue) and 2.5\%, 97.5\% quantiles (purple) from Table 4 marked. Location of PPARGC1A is marked in red. Rs8192678 is marked by a red dashed line. (ZIP $841 \mathrm{~kb}$ )

\section{Abbreviations}

BMI: Body mass index; CEU: Utah residents with Northern and Western European ancestry; CHB: Chinese in Beijing, China; CHS: Southern Han Chinese, China; Cl: Cook Island; GBR: British in England and Scotland; iHH: Integrated haplotype homozygosity; iHS: Integrated haplotype homozygosity score; NZ: New Zealand; SNP: Single nucleotide polymorphism; T2D: Type 2 diabetes; WGS: Whole genome (re)sequence; XP-EHH: Cross population extended haplotype homozygosity; YRl: Yoruba in Ibadan, Nigeria

\section{Acknowledgements}

This work was supported by contract C04X0804 from the New Zealand Ministry of Business, Innovation and Enterprise, and by the New Zealand Health Research Council. PW was also supported by a sabbatical research fellowship from New Zealand Forest Research Institute Limited. Supercomputing resources provided by the New Zealand National eScience Infrastructure (NeSl, see https://www.nesi.org.nz/) were used for running analytical pipeline. The authors also acknowledge helpful comments on an earlier version of the manuscript made by an anonymous reviewer.

\section{Funding}

This research was funded by Health Research Council of New Zealand (data generation) and the New Zealand Ministry of Business Innovation and Enterprise via contract C04X0804 (data analyses and interpretation).

\section{Availability of data and materials}

The datasets used and/or analysed during the current study are available from the corresponding author upon reasonable request.

\section{Authors' contributions}

MC carried out the genotyping, selection analyses and drafting of the manuscript. JB helped with the selection analysis and drafting of the manuscript. PW and TM conceived the study and participated in the design, interpretation of results, and coordination and helped draft the manuscript. MB participated in the statistical analysis and drafting of the manuscript. ND, LS and PV contributed samples and to the drafting of the manuscript. All authors read and approved the final manuscript. 


\section{Competing interests}

The authors declare that they have no competing interests.

\section{Consent for publication}

NA. There is no requirement for consent to publish from neither the researchers host organisation nor funder nor any other organisation.

\section{Ethics approval and consent to participate}

Ethical approval was obtained from the New Zealand Multi-region Ethics committee (MEC/105/10/130). Each participant provided informed written consent.

\section{Author details}

'Department of Biochemistry, University of Otago, Dunedin, New Zealand. ${ }^{2}$ Virtual Institute of Statistical Genetics (www.visg.co.nz), Dunedin, New Zealand. ${ }^{3}$ formerly Scion (New Zealand Forest Research Institute Ltd), 49 Sala

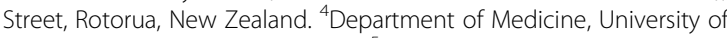
Auckland, Auckland, New Zealand. ${ }^{5}$ Department of Medicine, University of Otago, Christchurch, New Zealand. ${ }^{6}$ The Queensland Brain Institute, University of Queensland, Brisbane, Australia. ${ }^{7}$ University of Queensland Diamantina Institute, University of Queensland, Translational Research Institute (TRI), Brisbane, Australia. ${ }^{8}$ Department of Mathematics and Statistics, University of Otago, Science III Building, 730 Cumberland St, Dunedin 9016, New Zealand.

Received: 20 October 2015 Accepted: 1 November 2016 Published online: 15 November 2016

\section{References}

1. Neel JV. Diabetes mellitus: a "thrifty" genotype rendered detrimental by "progress"? Am J Hum Genet. 1962;14:353-62.

2. Kratzer JT, Lanaspa MA, Murphy MN, Cicerchi C, Graves CL, Tipton PA, et al. Evolutionary history and metabolic insights of ancient mammalian uricases. Proc Natl Acad Sci U S A. 2014;111:3763-8.

3. Handschin C. The biology of PGC-1a and its therapeutic potential. Trends Pharmacol Sci. 2009:30:322-9.

4. Myles S, Lea RA, Ohashi J, Chambers GK, Weiss JG, Hardouin E, et al. Testing the thrifty gene hypothesis: the Gly482Ser variant in PPARGC1A is associated with BMI in Tongans. BMC Med Genet. 2011;12:10.

5. Myles S, Hradetzky E, Engelken J, Lao O, Nürnberg P, Trent RJ, et al. Identification of a candidate genetic variant for the high prevalence of type II diabetes in Polynesians. Eur J Hum Genet. 2007:15:584-9.

6. Sundborn G, Metcalf PA, Gentles D, Scragg R, Dyall L, Black P, et al. Overweight and obesity prevalence among adult Pacific peoples and Europeans in the Diabetes Heart and Health Study (DHAHS) 2002-2003, Auckland New Zealand. N Z Med J. 2010;123:30-42.

7. Winnard D, Wright C, Taylor WJ, Jackson G, Te Karu L, Gow PJ, et al. National prevalence of gout derived from administrative health data in Aotearoa New Zealand. Rheumatology. 2012;51:901-9.

8. Winnard D, Wright C, Jackson G, Gow P, Kerr A, McLachlan A, et al. Gout, diabetes and cardiovascular disease in the Aotearoa New Zealand adult population: co-prevalence and implications for clinical practice. N Z Med J. 2013;126:53-64

9. Merriman TR. Population heterogeneity in the genetic control of serum urate. Semin Nephrol. 2011:31:420-5.

10. Cadzow M, Boocock J, Nguyen HT, Wilcox P, Merriman TR, Black MA. A bioinformatics workflow for detecting signatures of selection in genomic data. Front. Genet. 2014;5:1-8.

11. Hollis-Moffatt J, Phipps-Green A, Chapman B, Jones G, van Rij A, Gow P, et al. The renal urate transporter SLC17A1 locus: confirmation of association with gout. Arthritis Res Ther. 2012;14:R92.

12. Wallace SL, Robinson H, Masi AT, Decker JL, Mccarty DJ, Yü TF. Preliminary criteria for the classification of the acute arthritis of primary gout. Arthritis Rheum. 1977:20:895-900

13. 1000 Genomes Project FTP. 2015. ftp://ftp.1000genomes.ebi.ac.uk/vol1/ftp/ release/20130502/. Accessed 18 Aug 2015

14. Kimura R, Ohashi J, Matsumura Y, Nakazawa M, Inaoka T, Ohtsuka R, et al. Gene flow and natural selection in oceanic human populations inferred from genome-wide SNP typing. Mol Biol Evol. 2008;25:1750-61.
15. R Core Team. R: A language and environment for statistical computing. R Foundation for Statistical Computing, Vienna, Austria. 2015. URL https:// www.R-project.org/.

16. Graffelman J. Exploring Diallelic Genetic Markers: The \{HardyWeinberg\} Package. J Stat Softw. 2015;64:1-22.

17. Weir BS, Cockerham CC. Estimating F-Statistics for the Analysis of Population Structure. Evolution (N Y). 1984;38:1358-70.

18. Voight BF, Kudaravalli S, Wen X, Pritchard JK. A map of recent positive selection in the human genome. PLoS Biol. 2006;4:e72.

19. Tang K, Thornton KR, Stoneking M. A new approach for using genome scans to detect recent positive selection in the human genome. PLoS Biol. 2007;5:e171

20. Tajima F. Statistical method for testing the neutral mutation hypothesis by DNA polymorphism. Genetics. 1989;123:585-95.

21. Fay JC, Wu Cl. Hitchhiking under positive Darwinian selection. Genetics. 2000;155:1405-13.

22. Szpiech ZA, Hernandez RD. Selscan: an efficient multithreaded program to perform EHH-based scans for positive selection. Mol Biol Evol. 2014;31:2824-7.

23. Phipps-Green AJ, Hollis-Moffatt JE, Dalbeth N, Merriman ME, Topless R, Gow PJ, et al. A strong role for the ABCG2 gene in susceptibility to gout in New Zealand Pacific Island and Caucasian, but not Māori, case and control sample sets. Hum Mol Genet. 2010;19:4813-9.

24. Vitti JJ, Grossman SR, Sabeti PC. Detecting natural selection in genomic data. Annu Rev Genet. 2013:47:97-120.

25. Xue Y, Zhang X, Huang N, Daly A, Gillson CJ, MacArthur DG, et al. Population Differentiation as an Indicator of Recent Positive Selection in Humans: An Empirical Evaluation. Genetics. 2009;183:1065-77.

26. Bierne N, Roze D, Welch JJ. Pervasive selection or is it ...? why are F ST outliers sometimes so frequent? Mol Ecol. 2013;33:2061-4

27. Ferrer-Admetlla A, Liang M, Korneliussen T, Nielsen R. On detecting incomplete soft or hard selective sweeps using haplotype structure. Mol Biol Evol. 2014;31:1275-91.

28. Fu W, Akey JM. Selection and adaptation in the human genome. Annu Rev Genomics Hum Genet. 2013;14:467-89.

29. Ségurel L, Austerlitz F, Toupance B, Gautier M, Kelley JL, Pasquet P, et al. Positive selection of protective variants for type 2 diabetes from the Neolithic onward: a case study in Central Asia. Eur J Hum Genet. 2013:21:1146-51.

30. Lohmueller KE, Pearce CL, Pike M, Lander ES, Hirschhorn JN. Meta-analysis of genetic association studies supports a contribution of common variants to susceptibility to common disease. Nat Genet. 2003;33:177-82.

31. Ayub Q, Moutsianas L, Chen Y, Panoutsopoulou K, Colonna V, Pagani L, et al. Revisiting the thrifty gene hypothesis via 65 loci associated with susceptibility to type 2 diabetes. Am J Hum Genet. 2014;94:176-85.

32. Buck PH. The Coming of the Māori by Te Rangi Hiroa. New Zealand: Thomas Avery \& Sons; 1929.

33. Stafford DM. Te Arawa: A history of the Arawa people. Reed; 1967.

34. Kayser M, Brauer S, Cordaux R, Casto A, Lao O, Zhivotovsky LA, et al. Melanesian and Asian origins of Polynesians: mtDNA and Y chromosome gradients across the Pacific. Mol Biol Evol. 2006;23:2234-44.

35. Cagliani R, Sironi M. Pathogen-driven selection in the human genome. Int J Evol Biol. 2013:2013:1-6.

\section{Submit your next manuscript to BioMed Central and we will help you at every step:}

- We accept pre-submission inquiries

- Our selector tool helps you to find the most relevant journal

- We provide round the clock customer support

- Convenient online submission

- Thorough peer review

- Inclusion in PubMed and all major indexing services

- Maximum visibility for your research

Submit your manuscript at www.biomedcentral.com/submit
C) Biomed Central 